\title{
Epigenetic mechanisms and implications in tendon inflammation (Review)
}

\author{
FINOSH G. THANKAM ${ }^{1}$, CHANDRA S. BOOSANI ${ }^{1}$, MATTHEW F. DILISIO ${ }^{2}$ and DEVENDRA K. AGRAWAL ${ }^{1}$ \\ Departments of ${ }^{1}$ Clinical and Translational Science, and ${ }^{2}$ Orthopedic Surgery, \\ Creighton University School of Medicine, Omaha, NE 68178, USA
}

Received January 31, 2018; Accepted September 18, 2018

DOI: $10.3892 / \mathrm{ijmm} .2018 .3961$

\begin{abstract}
Cellular inflammation is not just an immediate response following pathogenic infections or resulting from damage due to injury, it is also associated with normal physiological functions, including wound healing and tissue repair. The existence of such a definitive role in normal physiology and in disease pathology indicates the presence of a regulatory mechanism that is tightly controlled in normal cells. A tight control over gene expression is associated with regulatory mechanisms in the cells, which can be either inducible or epigenetic. Among other intracellular mechanisms that contribute to epigenetic gene regulation, DNA methylation has been shown to maintain a tight control over gene expression through the actions of DNA methyltransferases (DNMTs). With a clear role in developmental and tissue-specific temporal gene regulation, the involvement of DNMTs is evident in normal and pathological conditions. In this review article, inflammation in tendons associated with disease pathology and tissue repair or regeneration at the musculoskeletal joints is critically reviewed. More specifically, the review focuses on known epigenetic mechanisms and their role in the clinical presentation of the disease in human joint disorders associated with tendon inflammation, with an emphasis on the gene regulatory mechanisms that are controlled through DNA methylation, histone deacetylation, and microRNAs.
\end{abstract}

\section{Contents}

1. Introduction

2. DNA organization and epigenetics

3. DNA methylation

Correspondence to: Dr Devendra K. Agrawal, Department of Clinical and Translational Science, Creighton University School of Medicine, CRISS II Room 510, 2500 California Plaza, Omaha, NE 68178, USA

E-mail:dkagr@creighton.edu

Key words: rotator cuff injury, inflammation, epigenetics, DNA methylation, histone modifications, RNA interference
4. Histone modifications

5. RNA-mediated epigenetics

6. Tendon disorders and epigenetic regulation of inflammation

7. Summary and future directions

\section{Introduction}

The response to inflammation constitutes a series of immune events that are evoked as a result of an insult in the tissues and aims to restore or re-establish normal structure and function. Inflammation is characterized by an increased local concentration of mediators, including inflammatory cells and cytokines (1). Chronic inflammation is a hallmark for several diseases, including cancer, diabetes, cardiovascular and pulmonary disorders, and neurological diseases. Furthermore, inflammation is associated with musculoskeletal insults, among which tendon inflammation in rotator cuff injury (RCI) is of particular concern (2). Persistent inflammation in the shoulder tendons delays the healing responses. Therefore, the management of inflammation and relieving of associated pain are required for a promising therapeutic approach.

Current knowledge of the molecular mechanisms and the primary cause of RCI associated inflammation remains limited. It is suggested that the physiological and mechanical stress in rotator cuff tendons results in microtrauma, which in turn leads to inflammation (3). Apart from the biochemical and molecular signals and pathways, epigenetic mechanisms are also involved in the initiation, progression, onset and regulation of inflammatory responses. Epigenetics-based therapeutic targeting of cellular or tissue inflammation in RCI has not been developed. Understanding the basics of epigenetic phenomena and the regulatory signaling is likely to be useful to elucidate the inflammatory mechanisms in RCI, the exploitation of which can assist in designing novel therapeutic approaches for RCI-associated inflammation.

\section{DNA organization and epigenetics}

Chromatin remodeling is an inevitable process that modulates and regulates gene expression. The structural dynamics of chromatin organization exposes the DNA strand for gene expression/regulation machinery. Chromatin exists as a complex of DNA, histones and non-histone proteins, which 
are organized into a specific 3D architecture that undergoes reversible and dynamic alteration during replication and gene expression. In addition, non-mutational structural modifications in DNA, including methylation and histone modifications, can alter gene expression which constitutes the epigenetic regulation system. The term 'epigenetics' was coined by Waddington in 1968 and was referred to as 'the interactions between genes and their products which bring phenotype into being' (4). Understanding the basic organization of the DNA in chromatin is necessary for examining the mechanisms of epigenetics involved in regulating gene expression.

The nucleosome is the basic unit of chromatin, which comprises $\sim 146$ base pairs (bp) of DNA wrapped around an octamer of histone protein subunits. These subunits include two copies each of H2A, H2B, H3 and H4. Nucleosomal organization ensures tight packing of the histones with DNA and enhances folding and stacking of otherwise lengthy DNA inside the nucleus. Therefore, nucleosome organization and chromatin condensation impede gene expression, which marks the remodeling of chromatin in the initial event of gene activation. The N-terminal ends of histones are susceptible to enzymatic reactions which facilitate local unwinding/rewinding of the nucleosome and have profound effects on gene expression (5). Linker DNA, which is between 10 and 80 bp in length, connects adjacent nucleosomes which are folded as a compact fiber of $30 \mathrm{~nm}$; these are further stacked to form higher order structures, including chromosomes. The nucleosomal organization of DNA is shown in Fig. 1. Linker histones (H1) are presumed to stabilize the 30 -nm fibers by binding to linker DNA. However, this interaction between $\mathrm{H} 1$ and linker DNA is debated, and the packing of DNA to its higher order structures within the nucleus remains to be fully elucidated. In short, these epigenetic mechanisms modulate gene function through DNA-protein interactions without altering the genetic code.

As compatible chromatin (heterochromatin) makes DNA inaccessible for gene expression machinery, the events that induce structural alterations in chromatin are crucial for the initiation of gene expression. However, actively expressing genes (euchromatin) are more susceptible for the action of specific enzymes and regulatory proteins involved in gene expression $(6,7)$. The transient modifications in chromatin structure are facilitated by remodeling complexes which shuffle nucleosomes to randomly expose the wound DNA for a limited time (7). Chromatin remodeling complexes can be ATP-dependent, which can move nucleosome positions to induce a conformational change to enable the DNA accessible on the histone surface. This complex mediates ATPase activity for energy and is predominantly associated with members of the SWI/SNF family. SWI/SNF subfamilies with helicase domains have also been established as epigenetic modulators. Remodeling complexes act by disrupting chromatin structure and by inducing covalent modifications to the nucleosomes through acetylation and methylation, with methylation occurring particularly at the histone $\mathrm{N}$-termini. These remodeling complexes are selective to the genes to be expressed and the transcription factors to be recruited during gene expression (8).

Mammalian epigenetic systems include DNA methylation, histone modifications and RNA interference. These systems can act individually, autonomously or cooperatively and can persist throughout the cell cycle, including during mitosis/meiosis. Disruption of these mechanisms may lead to the loss of cellular integrity, alterations in phenotype and disease progression, and impairments in normal development (9). Large-scale genome sequence variation (insertions, deletions or chromosomal rearrangements) ranging between $1 \mathrm{kbp}$ and several mbps, collectively termed copy number variants (CNVs), is also considered to be similar to epigenetic phenomenon $(10,11)$.

\section{DNA methylation}

DNA methylation is crucial for the normal development mediated through genomic imprinting, $\mathrm{X}$ chromosome inactivation, transcriptional repression and transposition. The failure to impart appropriate methylation tags to DNA contributes to genetic diseases and cancer (12). DNA methylation is catalyzed by DNA methyl transferases (DNMTs) with S-adenosyl methionine (SAM) as a methyl group donor $(13,14)$. DNMTs transfer the methyl group from SAM to cytosine residues on DNA, specifically at the $\mathrm{CpG}$ dinucleotide sequence yielding a 5-methylcytosine residue (Fig. 2A). In this regard, methylation in the promoter regions of the genes implies the extent of gene repression (15). The functional outcomes of DNMTs are appreciated in $\mathrm{X}$ chromosome inactivation and genomic imprinting in addition to the abnormalities in DNA methylation events which are associated with disease complications (16). Among the DNMTs (DNMT1, DNMT3A, and DNMT3B), DNMT1 is important as it prefers hemi-methylated DNA strands (17). DNMT1 is closely associated with newly replicated DNA, suggesting its function to methylate daughter strands with respect to the methylation patterns of the parental strand (18). DNMT3A and DNMT3B are de novo methyltransferases which act during development and are subsequently replaced with DNMT1 as cell division progresses (19). DNMT3A and DNMT3B usually act on unmodified DNA and cause hemi-methylation, whereas DNMT1 acts on hemi-methylated DNA. The representation of hemi-methylation and methylation patterns is shown in Fig. 2B.

DNMT3B is functionally active during early stages of embryogenesis and is known to arrest germ line genes during the transition from blastocyst to epiblast following implantation. By contrast, DNMT3A facilitates the retention and establishment of parental imprints and in differentiating somatic cell DNA methylation. Collectively, DNMT3A and DNMT3B maintain symmetry in CpG methylation, particularly at the hemi-methylated sites of embryonic cells $(20,21)$. DNMT3L, another DNMT, shares structural similarities with DNMT3A and DNMT3B with regards to the ATRX-DNMT3DNMT3-like domain and binds to the lysine $(\mathrm{K})$ residue at the 4th position of unmethylated H3. Although DNMT3L lacks catalytic activity, it is reported to function as a cofactor for DNMT3A and DNMT3B (22).

Unlike humans, mouse embryonic cells are reported to exhibit methylated genomic DNA at $\mathrm{CpA}, \mathrm{CpT}$ and $\mathrm{CpG}$ dinucleotides, with $\mathrm{CpG}$ being the predominant form in all somatic cells $(23,24)$; this shows that $\mathrm{CpG}$ methylation in the genome is common in the mammalian system. 5-methyl cytosine $(5 \mathrm{MeC})$ accounts for almost $1 \%$ of the total DNA bases of the genome, which constitute $>70 \%$ of all $\mathrm{CpG}$ dinucleotides. Those stretches of genomic sequences 


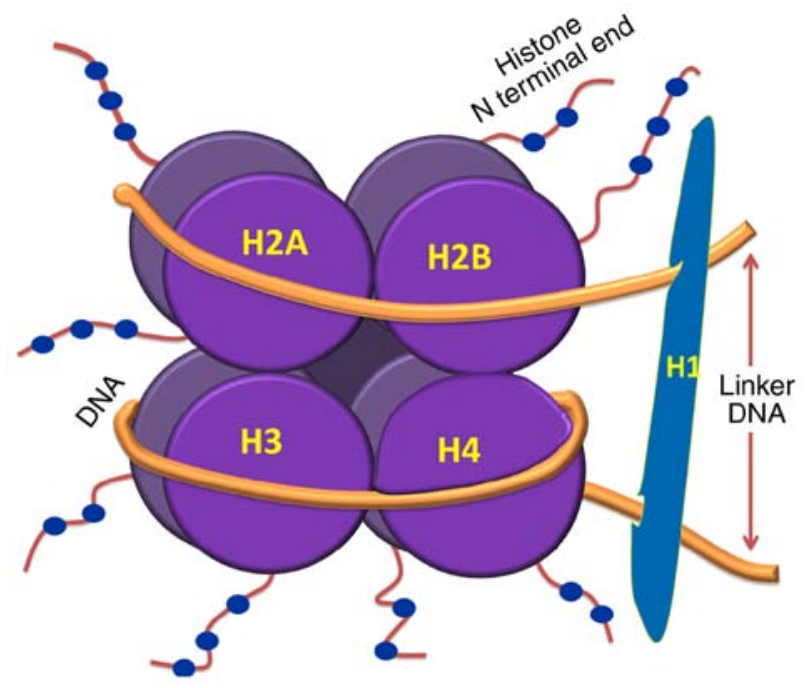

Figure 1. Nucleosomal organization of DNA. The DNA strand is wound around an octamer of histone subunits and the linker DNA is stabilized by linker histone $\mathrm{H} 1$.

comprising predominantly $\mathrm{CpG}$ dinucleotides concentrated in the genomic DNA are referred to as $\mathrm{CpG}$ islands, where the DNA is heavily methylated. These $\mathrm{CpG}$ islands are located around the promoter regions of several genes, signifying a role in gene regulation through the methylation of those genes. Due to the potential mutation resulting from the deamination of methylated cytosine to thymine, the majority of $\mathrm{CpG}$ dinucleotides are confined to $\mathrm{CpG}$ islands (25). The methylated state of DNA is the result of dynamic, but independent, methylation and demethylation, which varies between cell types. For example, mature germ cells and somatic cells are heavily methylated compared with hypomethylated embryonic cells $(26,27)$. The non-X-linked promoter $\mathrm{CpG}$ islands are reported to be methylated in normal tissues but exempt from methylation in germ line cells. DNA or histone methylation recruits repressive machinery and elicits an unfavorable chromatin conformation to prevent transcription (28). Such alterations in chromatin structure induce methylation of the adjacent chromatin segments (29). In addition, the methylation at $\mathrm{H} 3 \mathrm{~K} 4$ assists in the assembly of regulatory proteins of the chromatin-remodeling complex which offers transcriptional regulation. The mechanism of the regulatory role of H3K4 methylation is described in detail in the following sections.

Transcriptional repression by DNA methylation. $\mathrm{CpG}$ islands are associated with promoter regions of the majority of housekeeping genes and other genes that are regulated during developmental stages. Such loci are transcriptionally inactive irrespective of their hypomethylation status (30). The hypomethylated state is necessary for the binding of transcription factors (TFs), which prevents DNA methylation and histone methylation machinery from targeting these loci $(31,32)$. Based on the transcriptional activity of the $\mathrm{CpG}$ islands, the associated promoters can be poorly methylated, including intermediate $\mathrm{CpG}$ density promoters (inactive on methylation) and low $\mathrm{CpG}$ density promoters (hypermethylated), or transcriptionally active promoters regardless of their methylation status (31). The intermediate $\mathrm{CpG}$ density promoters can attain
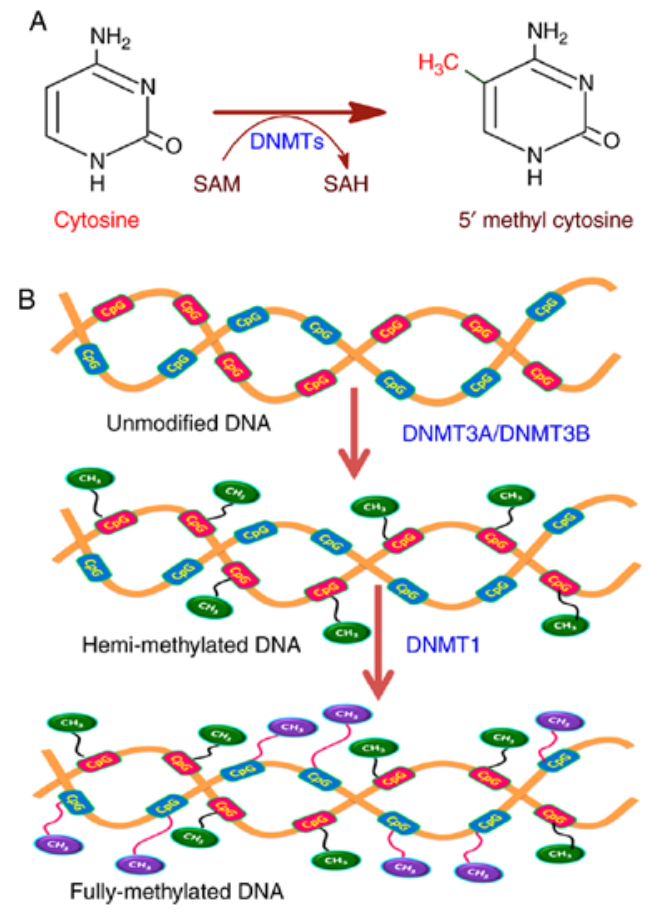

Figure 2. Activity of DNMTs. (A) DNMT catalyzes the transfer of methyl group from SAM to the 5th carbon of cytosine to form 5'-methyl cytosine. (B) Unmethylated genomic DNA is acted on by DNMT3A and DNMT3B to form hemi-methylated DNA, which is converted to fully-methylated DNA by the activity of DNMT1. SAM, S-adenosyl methionine; SAH, S-adenosylhomocysteine; DNMT, DNA methyl transferase.

hypermethylation upon encountering differentiation signals. This DNA methylation offers an effective method of specific gene silencing, which was observed during the differentiation of germ cells (20). As with promoters, enhancer methylation can also modulate gene expression, however, the regulatory role of enhancer methylation remains to be elucidated (33).

The level of DNMT1 is reported to be increased during the $\mathrm{S}$ phase of the cell cycle, as evident from its high expression in mitotic cells. Proliferating cell nuclear factor-interacting binding factor and nuclear protein 95 attract DNMT1 to the replication fork to ensure its binding to the unmethylated daughter strand, converting hemi-methylated DNA into fully methylated DNA (34). The resulting methyl groups of $5 \mathrm{MeC}$ occupy the major groove of the DNA duplex and inhibit transcription by preventing TF binding or by interacting with methyl-binding proteins which recruits transcriptional repressors (35). In addition, the presence of methylated DNA elements within the genes facilitates the elongation phase of transcription and minimizes premature initiation (36). In addition, splicing of the transcripts is favored by methylation as the exon-intron boundaries are characterized by transitions in methylation pattern (37).

Demethylation. The reprogramming of $5 \mathrm{MeC}$ to reset the methylation status of cells for each new generation is essential, which suggests the existence of cellular demethylation machinery. The zygote presents DNA demethylation as compartmentalized deletion of $5 \mathrm{MeC}$ with maternal and paternal chromosomes leading to a demethylated epigenome. However, imprinted gene loci, certain maternally-contributed 
promoters, and several transposable elements (TEs) are exceptions (20). It has been reported that the maternal genome retains $5 \mathrm{MeC}$ and depletes during subsequent cell cycles, whereas the paternal genome demethylates prior to cell division (38). However, it has been reported that $5 \mathrm{MeC}$ is globally converted to 5 '-hydroxymethyl cytosine $(5 \mathrm{hMeC})$, which is removed in subsequent replications (39). In addition, a second stage of reprogramming of DNA methylation occurs in primordial germ cells to decrease global $5 \mathrm{MeC}$ levels. The regulation of gene expression in a locus-specific manner has also been revealed. Reprograming by demethylation is also vital for cell fusion and the differentiation of induced pluripotent stem cells (40).

Several proteins that exhibit demethylase activities have been reported in the mammalian system. The ten-eleven translocation (TET) family of deoxygenases have been found to catalyze the conversion of $5 \mathrm{MeC}$ to $5 \mathrm{hMeC}$ and then 5'-formyl cytosine $(5 \mathrm{FC})$ and finally 5 -carboxyl cytosine $(5 \mathrm{CaC})$ in three sequential oxidation reactions. Subsequently, $5 \mathrm{CaC}$ is decarboxylated to cytosine resulting in demethylation. TET belongs to the $\mathrm{Fe}(\mathrm{II}) / \alpha$-ketoglutarate-dependent dioxygenases, which utilize $\mathrm{Fe}(\mathrm{II})$ as cofactor (41). TET has been identified in acute myeloid leukemia as part of histone H3 Lys 4 (H3K4). Of the three members in the TET family, TET1-3, TET3 possesses $5 \mathrm{MeC}$ hydroxylase activity. TET activity modifies the existing DNA methylation pattern, which paves the way for transcriptional regulation (42). The TET-mediated demethylation of $5 \mathrm{MeC}$ is shown in Fig. 3.

\section{Histone modifications}

Post-translational modifications in histones, particularly at the specific amino acid residues of $\mathrm{N}$-terminal ends, have implications on the extent of gene expression. A wide array of modification patterns have been established on histones (Table I) where the acetylation/deacetylation and methylation of $\mathrm{K}$ residues are of significance in terms of transcriptional regulation $(43,44)$ (Fig. 4). In general, acetylation facilitates the decondensation of chromatin, which exposes the genes to enable access to the replication/transcription machinery, and deacetylation causes the chromatin condensation that represents inactive genes. The acetylation neutralizes the negative charge density around the genes and disturbs the secondary structure of DNA, which makes the genes accessible for interacting with regulatory signals. In short, the interplay between acetylation and deacetylation determines the regulation of gene activity (45). The gene regulation by histone methylation depends on the amino acid residue undergoing methylation (16). Similarly, histone modifications caused by phosphorylation can either activate or inactivate the genes with respect to the inducing signals. The actual effects of other histone modifications on gene expression remain to be fully elucidated.

The combinations of histone modifications at a promoter region can determine the epigenetic status of cells, which facilitates either gene activation or repression and constitutes the 'histone code' hypothesis. The molecular cues for these modifications are encoded in the tail domains of histones, which are read by the regulator molecules. The parental histones are distributed randomly on daughter strands

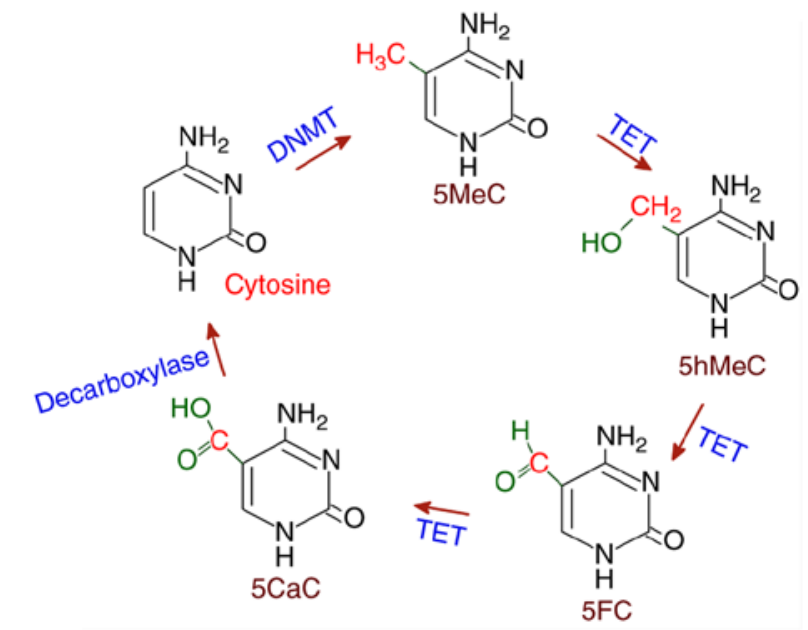

Figure 3. Demethylation reaction by TET. The $5 \mathrm{MeC}$ is converted to $5 \mathrm{hMeC}$, $5 \mathrm{FC}$ and finally to $5 \mathrm{CaC}$ by sequential oxidation by $\mathrm{TET}$, and $5 \mathrm{CaC}$ is decarboxylated to cytosine by the decarboxylase reaction. TET, ten-eleven translocation; $5 \mathrm{MeC}, 5$-methyl cytosine; $5 \mathrm{hMeC}, 5$ '-hydroxymethyl cytosine; 5FC, 5'-formyl cytosine; 5CAC, 5'-carboxyl cytosine; DNMT, DNA methyl transferase.

following replication and retain an epigenetic memory for gene expression (46).

The enzymes that catalyze histone acetylation, mainly at $\mathrm{K}$ residues, can be collectively referred to as histone acetyl transferases (HATs). The enzymes that remove acetyl groups from acetylated histones are histone deacetylases (HDACs). HATs are usually associated with transcriptional activators, whereas HDACs form part of transcriptional repressors. In short, the balance between HAT and HDAC activities are significant for the epigenetic regulation of gene expression. $\mathrm{K}$ and arginine $(\mathrm{R})$ residues are subjected to methylation by histone methyltransferases (HMTs). The methylation at K residues is generally considered as a stable and readily reversible histone modification compared with others. The existence of histone demethylases (HDMs) has also been reported (45). Transcriptionally active genes exhibit acetylation/methylation of K4, K36 and K79 of H3 and vice-versa. The majority of the $\mathrm{K}$ residues subjecting to HAT activity reside in the N-terminal tail of histone, although H3K56 in the core domain is an exception; the acetylated H3K56 residue exists in the core region of the $\mathrm{H} 3$ subunit (43). In addition, certain HMTs have also been found to be associated with HATs. For example, coactivator-associated arginine methyltransferase CARM1/PRMT4 is attached to HAT with physical interactions and acts cooperatively in mediating nuclear factor $-\kappa \mathrm{B}$ (NF- $\mathrm{kB}$ ) activity (47). G9a, a histone lysine methyltransferase, also has a dual function as a transcriptional suppressor and activator (48).

The role of histone modifications is also central in responses to DNA damage for marking the damage site. For example, the methylation of H4K20 and action of cell cycle checkpoint protein $\mathrm{Crb} 2$ result in cell cycle arrest at the $\mathrm{G} 2 / \mathrm{M}$ transition (49). Histone acetyltransferase binding to origin recognition complex HBO1 assists in chromatin remodeling during replication by coordinating with the ING family of tumor suppressors. $\mathrm{H} 4$ acetylation by $\mathrm{HBO} 1$ and the association of ING4, ING5 and p53 in the protein complex containing 
Table I. Types of histone modification.

\begin{tabular}{ll}
\hline Histone modification & \multicolumn{1}{c}{ Residue } \\
\hline Acetylation & Lysine (K) \\
Methylation & Lysine (K) \\
Methylation & Arginine (R) \\
Ubiquitination & Lysine (K) \\
Phosphorylation & Serine (S) \\
Phosphorylation & Threonine (T) \\
ADP ribosylation & Glutamic acid (E) \\
Sumoylation & Lysine (K) \\
Deimination & Arginine (R) \\
Proline isomerization & Proline (P) \\
\hline
\end{tabular}

HBO1 are evident in S phase cells; the p53-HBO1 interaction occurs through physical binding and this interaction decreases the activity of HAT (50). Histone modification by phosphorylation/dephosphorylation is also significant in mammalian cell replication, mitosis, apoptosis and gametogenesis as these modifications can induce compaction/decompaction of DNA by altering the charge density (51).

Regulation of histone modifications. Histone modifications rely on unraveling chromosomal DNA and the recruitment and assembly of regulatory signals to the target site. Such regulatory signals possess catalytic domains, the activities of which can trigger a cascade of events that result in transcription, replication or repair depending on cellular status. The regulatory proteins associated with histone modifications are characterized by specific domains. For example, chromo-like domains of the Royal family recognize methylation and plant homeodomain (PHD), acetylation by bromodomain and phosphorylation by 14-3-3 domain (43). The recruitment of basal transcription machinery by proteins with HAT activities, including p300 and CREB-binding protein (CBP), and chromatin remodeling complex components, including Brahma-related gene-1 are facilitated by acetylated histones. These proteins bind to acetylated nucleosomes or acetylated histone chains with higher affinity. In addition, TATA box binding protein-associated factor 1 binds to acetylated histone and promotes the ubiquitination and phosphorylation of H1, by which transcription is activated (52).

Methylation at $\mathrm{H} 3 \mathrm{~K} 4$ assists in assembling regulatory proteins by binding to bromodomain PHD finger transcription factor via the PHD domain, a key component of the nucleosome remodeling factor chromatin-remodeling complex. This assembly is followed by the recruitment of sucrose non-fermenting homologue 2-like ATPase, resulting in activation of the homeobox C8 gene (53). Similarly, Jumonji domain (JMJD)2A, a lysine demethylase, and chromodomain helicase DNA binding protein 1, an ATPase, bind to methylated H3K4 with the tudor domain and chromodomain, respectively (54). The structural basis of these interactions remains to be fully elucidated and the binding is reported to occur preceding H3R2 (55). These protein domains act as adaptors for binding and a remodeling complex through methylated $\mathrm{H} 3$.
Methylated H3L27 recruits polycomb protein PC2, which exhibits ubiquitin ligase activity. Similarly, HP1 protein interacts with $\mathrm{H} 3 \mathrm{~K} 9$ and exhibits methyltransferase and deacetylase activities. The methylation of $\mathrm{H} 3 \mathrm{~K} 4$ and phosphorylation of H3T3 interfere with the binding of the transcriptionally repressive NuRD complex and inhibitor of acetyltransferase complex, respectively $(52,56)$. The binding of a protein to histone may also disrupt the modification in adjacent residues, which is evident from altered heterochromatin protein 1 (HP1) binding to methylated H3K9 upon the phosphorylation of H3S10 (51). In addition, a modification in one histone tail can alter the modification on a different histone tail. For example, the ubiquitination of $\mathrm{H} 2 \mathrm{~B}$ is required for the methylation of H3K4 (43). Suppressor of variegation 3-9 homolog 1 (SUV39H1) is a lysine methyltransferase recruited by trimethylated $\mathrm{H} 3 \mathrm{~K} 9$-bound HP1 that methylates adjacent histones. SUV39H1 activity facilitates further binding of HP1 resulting in opening of the heterochromatin region. Similarly, Enhancer of zeste homolog 2 possesses methyltransferase activity specific to $\mathrm{H} 3 \mathrm{~K} 27$ and recruits the dimeric protein $\mathrm{PC}$, which is a component of the polycomb repressive complex 1 . The binding of $\mathrm{PC}$ to histones prevents access of the SWI/SNF remodeling complex to chromatin, preventing transcription (57).

According to 'histone code hypothesis', histone modification at specific residues determines subsequent modifications in the same or a different histone (52). These modifications are recognized by regulatory proteins resulting in chromatin remodeling and transcription. For example, the methyl/phospho binary switch hypothesis states that the combination of several modifications results in the transition of a stable methyl/lysine state to a dynamic transcriptional state, and the phosphorylation of $\mathrm{S} / \mathrm{T}$ residues adjacent to methylation site channels the downstream proteins to facilitate transcription. This type of regulation by phosphorylation depends on the position of phosphorylated residues, if a phosphorylated residue occurs prior to methylation and transcription is activated and vice versa (52).

Investigations on yeast chromosome mutations have revealed the ubiquitination of $\mathrm{H} 2 \mathrm{~B}-\mathrm{K} 123$ occurred previous to $\mathrm{H} 3-\mathrm{K} 4$ and that $\mathrm{H} 3-\mathrm{K} 79$ methylation facilitates chromatin opening and transcription (58). In addition, the dynamics of H2B ubiquitination/de-ubiquitination determines the transcription of a certain set of genes. The de-ubiquitination activity of ubiquitin-specific processing protease 8 of Spt-Ada-Gcn5-acetyltransferase is essential for the methylation of H3-K36, which in turn results in the gene expression of galactokinase 1 in budding yeast (59). The proteosomal ATPases, regulatory particle triple-a protein, or regulatory particle triphosphatase (Rpt)4 and Rpt6 activate the methylation of H3-K4 and H3-K79 by altering the chromatin structure in the vicinity of ubiquitination (by Rad6). The $19 \mathrm{~S}$ proteasome component ATPases independent of 20S facilitates the initiation and elongation phases of transcription and the $20 \mathrm{~S}$ sub-complex is associated with RNA polymerase III $(60,61)$.

In higher eukaryotes, the impact of histone modifications on transcription is complex and contrasting effects exist in the modification patterns of histone residues. In addition, the actual role and effect of modifications including sumoylation, ubiquitination and de-ubiquitination remain to be fully 
A<smiles>CNC(CCCCN)C(=O)O</smiles>

Lysine residue at $\mathrm{N}$ terminal end of histone .

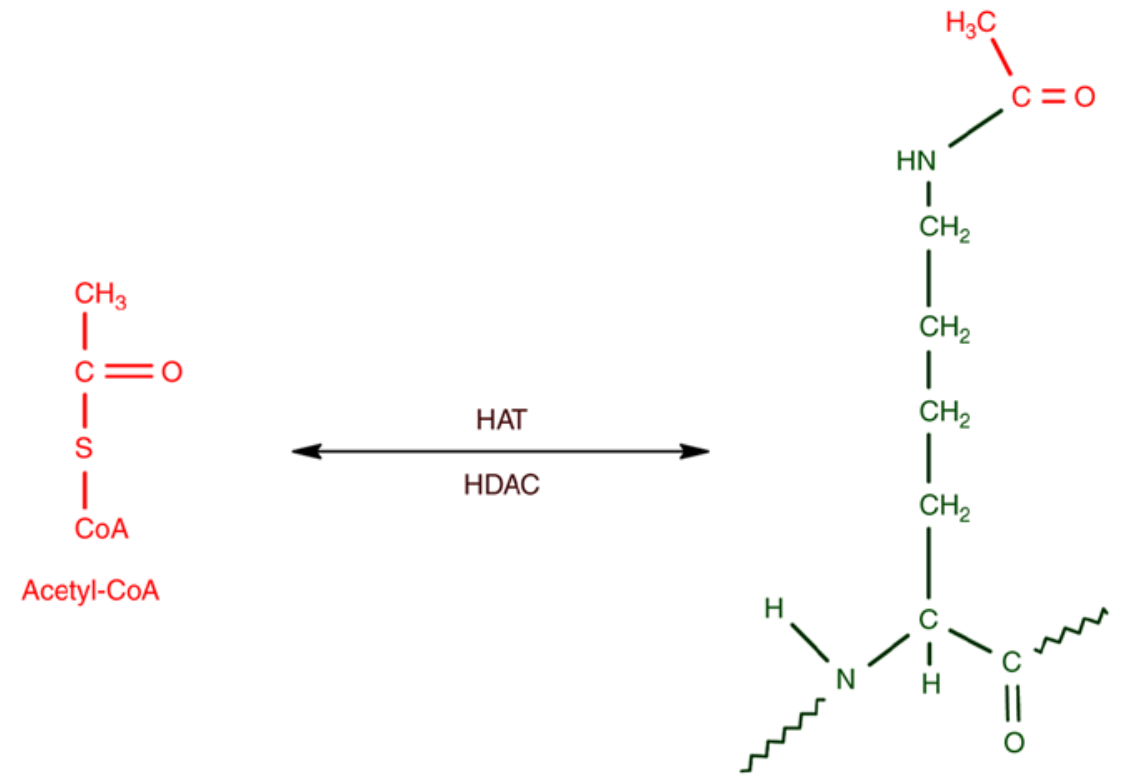

Acetylated histone

\section{B}

SET1
MLL/HRX/ALL1
MLL2/HRX2
MLL/HALR
ASH1
SMYD3


SET and MYND domain containing 3 (SMYD3), which also has a DNA binding domain, requires detailed investigation. SMYD3 can behave as a transcription factor and as a DNA binding enzyme, however, its epigenetic implications remain to be fully elucidated.

The epigenetics of histone ubiquitination in eukaryotes remains unclear, however, the ubiquitin conjugating enzyme Ubc2 facilitates the methylation of H3K4 by the ubiquitination of H2B-K123 in Saccharomyces cerevisiae. In addition, investigations of mutations have shown that the ubiquitination of H2B-K123 is a prerequisite for H3K4 methylation (62). The involvement of ubiquitination and SUMOylation in the epigenetic regulation of inflammatory responses remains to be fully elucidated, and there is scope for extensive investigation. The ubiquitination and SUMOylation of NF- $\mathrm{\kappa B}$ activates inflammatory responses, but not in an epigenetic manner $(64,65)$. Further investigation of these aspects in relation to inflammation and inflammatory disorders is warranted.

\section{RNA-mediated epigenetics}

The emergence of long non-coding RNAs and small non-coding RNAs through either intergenic or antisense transcription has been shown to be involved in regulating chromatin organization, translational repression and gene expression in eukaryotic cells. The small RNAs exert their effect via RNA interference (RNAi) pathways $(66,67)$. The implications of DNA methylation and subsequent gene silencing mediated through RNAi has been established in Caenorhabditis, Schizosaccharomyces, and Tetrahymena, and also in somatic and germ cells $(68,69)$. Elucidation of the RNAi-mediated downregulation of genes in human diseases offers potential for RNA-based therapeutic strategies. However, the selection of small interfering RNAs (siRNAs) or microRNAs (miRNAs), and knowledge of multiple targets and binding efficiency/strength are crucial to minimize unwanted reactions.

In general, small RNAs that regulate gene expression in the cytoplasm can be either miRNAs, which are derived from a hairpin loop, cause translational repression and usually carry non-complimentary sequences to the target (70); siRNAs, which cause the degradation of the transcript owing to its sequence complementarity; or piwiRNAs (piRNAs), which mediate transposon transcripts. These RNAs are capable of guiding chromatin modifications mediated through histone/DNA methyltransferases (71).

The biogenesis of siRNAs is predominantly elicited by double-stranded RNAs (dsRNAs) and is mediated by the Argonaute (Ago) family of proteins. Ago proteins are ubiquitous effectors for miRNAs and siRNAs whereas piwi proteins are responsible for piRNAs (72). The precursor dsRNA is cleaved by the Dicer family of enzymes, including RNAse III-forming 20-25 nucleotide (nt) duplexes where $3{ }^{\prime} \mathrm{OH}$ overhangs $5{ }^{\prime} \mathrm{PO}_{4}(73)$. Evidence for the existence of several Dicer-independent mechanisms of siRNA synthesis is also available in the literature (74). The cellular location of siRNA biogenesis is debated; however, a large body of evidence suggests that the cytoplasm is the possible site. By contrast, in S. pompe, Ago-mediated cleavage and RNA-dependent RNA polymerase amplification occurs in the nucleus (71). The generated duplexes are then loaded to the effector Ago protein, which requires heat shock protein 90 . However, the mechanism, location and regulation of these proteins in regard to RNAi remain to be elucidated. Gene silencing by the miRNA interference pathway is shown in Fig. 5.

The link between the RNAi pathway and nucleosome structure has been revealed by the identification of heterochromatic chromodomain protein calcineurin-like EF-hand protein 1 as a component of RNA-induced transcriptional silencing (RITS) associated with Agol and small RNAs. Trans-acting siRNA Tas3 associates with RITS through Ago1 and the functional RITS complex comprises an RNA-dependent RNA polymerase complex (RDRC; e.g. Rpd1), helicases (e.g. helicase required for RNAi-mediated heterochromatin assembly), and poly(A) polymerase CTC-interacting domain 12) (75). The RITS complex interacts with the heterochromatin and small RNAs, although the exact function and mechanism of this interaction remains to be elucidated; however, by binding to euchromatic mRNAs, RITS leads to the methylation of H3K9 (76). The RNAs associated with chromatin can act as scaffolds for the organization of RITS and assists in the recognition of chromatin modifications which ultimately lead to H3K9-mediated gene silencing (77).

The Clr4 subunit of the Clr4-Rik1-Cul4 (CLRC) complex, a protein complex that assembles at the target nucleosome, possesses methyltransferase activity which methylates H3K9. Rik1 links with the RDRC and RITS complex, and the Stclprotein of the CLRC interacts with Ago and Tas3, thereby linking the RNAi machinery (77). These interactions are reported to occur through physical means, which suggests feedback among the RITS complex, the RDRC and the CLRC complex (78,79). Rik1-associated factor 2 (also known as Cmc2) and Rik1, components of the CLRC complex, associated with Cdc20 (the catalytic subunit of the leading-strand DNA polymerase- $\varepsilon$ ) and Mms19 (a regulator of the TFIIH) coordinate DNA replication and the RNAi-mediated release of RNA polymerase II for heterochromatin-mediated inheritance $(80,81)$. These results were the outcome of studies performed in Saccharomyces species and the evidence for the coordination between RNAi and DNA replication requires detailed investigation, particularly in higher eukaryotes.

\section{Tendon disorders and epigenetic regulation of inflammation}

Tendon disorders, particularly rotator cuff tendinopathies, are one of the most common musculoskeletal disorders of which pain and inflammation are the major symptoms. Shoulder tendinopathies constitute $>30 \%$ of referrals for musculoskeletal disorders. Despite the increased incidence of tendinopathies, the exact mechanisms underlying the etiology and pathogenesis of shoulder tendinopathies remain to be fully elucidated (82). The expression of inflammatory cytokines and mediators associated with tendinopathies has been reported in several studies. Tenocytes upon inflammatory trigger/stimuli tend to express inflammatory cytokines, including tumor necrosis factor (TNF)- $\alpha$, interleukin (IL)-1 $\beta$, IL-6, IL-21, and transforming growth factor (TGF) $-\beta(83,84)$.

Of note, the shoulder tendon tissues in the majority of cases do not show the classical histology of inflammation, 


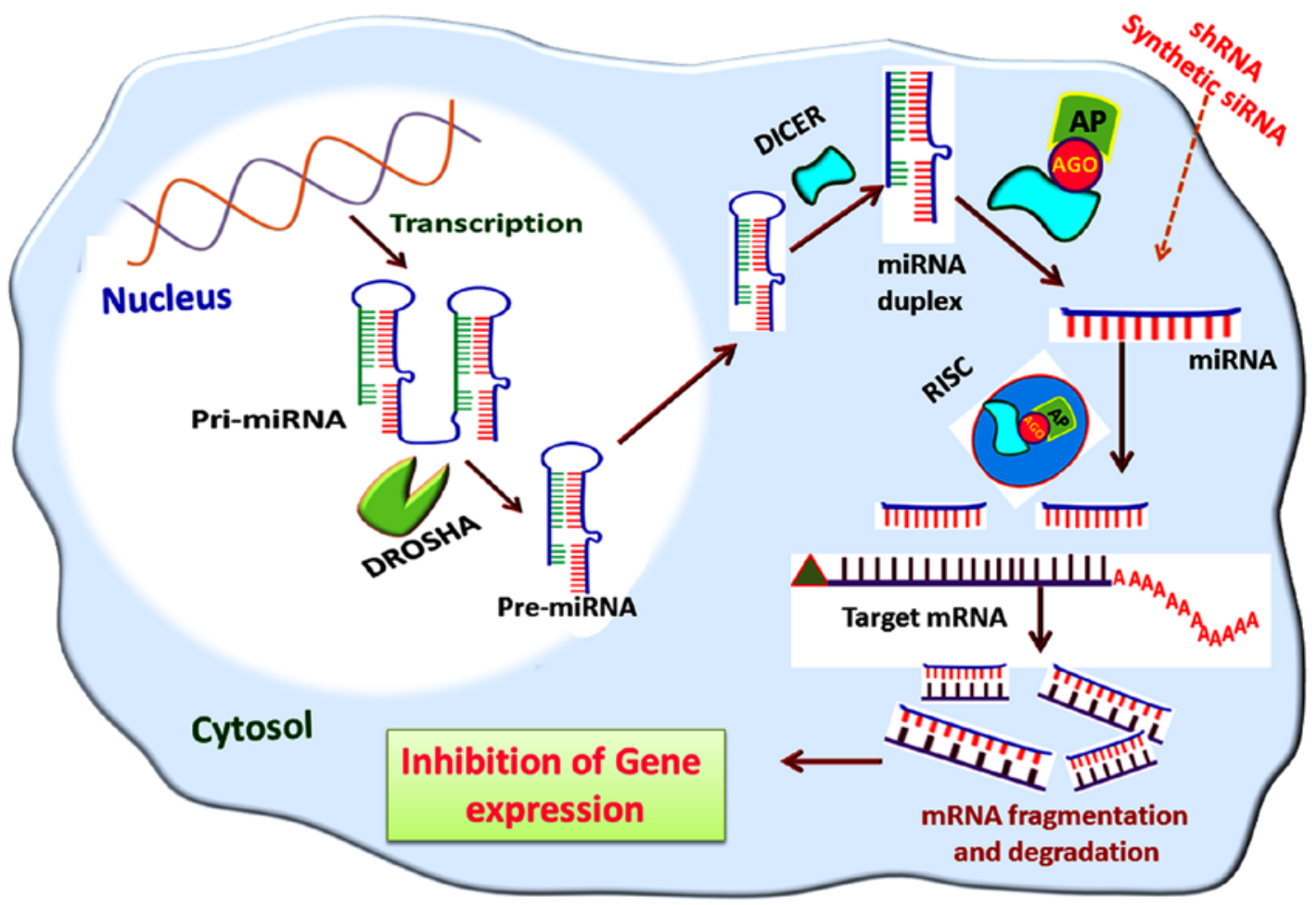

Figure 5. Gene silencing via the miRNA interference pathway. The pre-miRNA formed in the nucleus by DROSHA activity is transported to the cytosol mediated by exportin-5; in the cytosol, the pre-miRNAs are cleaved by nucleases, including DICER, to miRNA duplexes from which miRNAs are formed. The miRNAs are then assembled to the RISC and leads to the degradation of target mRNA transcripts. A similar mode of action is elicited by shRNA and synthetic siRNA, if delivered to the cytosol. miRNA, microRNA; pri-miRNA, primary miRNA; pre-miRNA, precursor miRNA; RISC, RNA-induced silencing complex; AGO, argonaute; AP, effector AGO protein; shRNA, short hairpin RNA; siRNA, small interfering RNA.

including the infiltration of macrophages or neutrophils and cytokine production (84). By contrast, reports showing the presence of the infiltration and activation of immune cells are also available; however, this depends on the severity of the injury (85). Our previous study described a novel dual-mechanism of the inflammatory status of injured rotator cuff tendon tissue in relation to triggering receptor expressed on myeloid cells-1 (TREM1). During asymptomatic tendinopathies, the tenocytes express the TREM1 molecule and function like immune cells, which in turn are regulated by high mobility group protein 1 and receptor for advanced glycation end products $(1,86)$.

So far, the epigenetics of inflammation associated with tendon disorders has not been investigated, however, the basic cellular mechanisms appear to be applicable to tenocytes. The trigger for the epigenetic switch and the pathological aspects of epigenetic alterations in tendon cells remain to be fully elucidated, however, several miRNAs have been reported to be involved in tendon disorders and inflammation (87). For example, microRNA (miR)-29a is involved in the regulation of IL-33-mediated inflammation in rotator cuff tendons (88). In our previous studies, the screening of alterations of miRNAs associated with shoulder tendon matrisome disorganization (89), and the Janus kinase 2/signal transducer and activator of transcription (STAT)3 pathway of inflammation (90) was performed. These studies revealed the alteration of hundreds of miRNAs which are considered to be associated with the pathological changes in the tendon. Among them, miR-145-5p, miR-100-5p, miR-195-5p, and let-7 were found to be the key miRNAs, and warrant further detailed investigations.

Due to the limited availability of literature regarding the epigenetic regulations of tendon inflammation, the following section mainly describes the epigenetics of general inflammation irrespective of specific tissue; the extrapolation of such knowledge to tendon tissues may have an impact on tendon disorders.

The inflammatory response is complex and is elicited by signal-specific or gene-specific cascade mechanisms which usually result in antimicrobial defense, immune response and repair, and regeneration of the affected tissue (91). A wide array of transcription factors associated with inflammatory responses, including NF- $\mathrm{NB}$, forkhead box P3 (FOXP3), and STAT2 are regulated by epigenetic changes including DNA methylation and/or histone modifications (92). For example, JMJD3 regulates the methylation status of $\mathrm{H} 3 \mathrm{~K} 27$ and controls the differentiation and phenotype identity of macrophage cells. The IL-4 mediated activation of JMJD3 removes the repressive methylation tag from H3K27 of the STAT6 promoter, which in turn activates the downstream inflammatory genes. In addition, activated STAT6 positively regulates JMJD3 by binding to its promoter (93).

The impairment of DNA methylation following chronic inflammation has led to an understanding of the role of the polycomb group proteins in the epigenetic regulation of inflammatory responses (94). NF- $\kappa \mathrm{B} /$ reticuloendotheliosis B (RelB)-mediated gene repression is another classical example. RelB induction, resulting from the actions of endotoxins or 
during sepsis, represses a number of proinflammatory genes by enhancing heterochromatin formation through interacting with H3K9 methyltransferase G9a. The subsequent trimethylation of $\mathrm{H} 3 \mathrm{~K} 9$ recruits $\mathrm{HP} 1$ to form a repressive complex at RelB promoters, and this recruits DNMT3a and DNMT3b to $\mathrm{CpG}$ methylation. Therefore, RelB links histone modifications and DNA methylations which have implications in inflammation $(95,96)$.

The activation of HAT results in the transcription of inflammatory genes whereas HDAC represses inflammation. Proinflammatory cytokines, including IL-1, IL-8, IL-2 and IL-12, are acetylated by $\mathrm{CBP} / \mathrm{p} 300$ at their promoter regions leading to their transcription. In addition, the activity of HDAC represses the transcription of these genes. Proteins with HDAC activity also regulate the transcription of proinflammatory and anti-inflammatory cytokines by recruiting co-repressors and transcription factors, including FOXP3, STATs, GATA, zinc finger E-box-binding homeobox 1 and NF- $\mathrm{B}$ (97). Following challenge with cytokines, $\mathrm{NF}-\kappa \mathrm{B}$ is regulated by inhibitor of $\mathrm{NF}-\kappa \mathrm{B}$ kinase subunit $\alpha(\mathrm{IKK}-\alpha)$ of inhibitor of NF- $\kappa \mathrm{B}(\mathrm{I} \kappa \mathrm{B})$ kinase, and forms a complex by binding with the promoter region. This binding is facilitated by the RNA polymerase II complex and CBP, where IKK- $\alpha$ promotes the acetylation of H3K 9 and phosphorylation of H3S10. The phosphorylation of H3S10 leads to CBP-dependent H3K14 acetylation, which channels $\mathrm{NF}-\kappa \mathrm{B}$ flux. HDAC and glucocorticoid receptor activation can reverse these processes leading to the transcriptional repression of NF- $\mathrm{NB}$-dependent inflammatory genes (98).

The epigenetic regulation of inflammatory genes by DNA methylation has also been reported. The hypomethylation of Toll-like receptor (TLR)2 results in the aggravation of inflammation, as reported in bronchial epithelial cells following challenge with bacterial peptidoglycan (99). The combined effects of DNA methylation and histone acetylation result in regulation of the TLR4 gene in the epithelial lining of the intestine, suggesting the role of epigenetic DNA methylation on microbial defense (100). The increased methylation of interferon- $\gamma$ promoter cells and the demethylation of IL- 2 and IL- 6 promoters on $\mathrm{CD}^{+}{ }^{+} \mathrm{T}$ cells upon allergen challenge on experimental asthma models provide further evidence $(101,102)$. Helicobacter pylori induces inflammation of the gastric mucosa and activates oncogenic pathways by altering the methylation patterns of gastric epithelial cells (103). In addition, the inflammatory cytokine TGF- $\beta$ suppresses CD133 stem cell/cancer biomarker by methylation, with demethylation at the promoter regions inducing resistance to apoptosis and anticancer drugs $(104,105)$. In addition, the hypermethylation of suppressor of cytokine signaling 1 in macrophages regulates cytokine signaling, particularly TNF- $\alpha$ and IL-6, and thereby inflammation $(106,107)$.

Chemokines of the (C-X-C motif) ligand (CXCL) family are reported to be involved in various carcinogenic and anti-carcinogenic signaling pathways and in inflammatory responses. CXCL1/growth regulated oncogene- $\alpha(\mathrm{GRO} \alpha)$ enhances angiogenesis and inhibits extracellular matrix (ECM) synthesis in prostate cancer cells. CXCL1/GRO $\alpha$ acts by the activation of $\mathrm{NF}-\kappa \mathrm{B}$ and its subsequent interaction with HDAC1 to repress the ECM protein, fibulin-1D (108). Similarly, the expression of CXCL14 is suppressed by the hypermethylation of $\mathrm{CpG}$, and this hypomethylation enhances its expression. CXCL14 exerts its anticancer function by preventing cell migration and invasion by inhibiting $\mathrm{NF}-\kappa \mathrm{B}$ signaling $(109,110)$. CXCL12 functions by binding to the receptor CXCR4, where it is downregulated by aberrant methylation in breast cancer cells. As the existence of demethylated CXCR4 and hypermethylated CXCL12 has been established in tumor cells, the methylation status of the cells can be used as a diagnostic tool for various types of cancer $(111,112)$.

\section{Summary and future directions}

The epigenetic regulation of gene expression has been established in mammalian systems, including humans. The structures, localization, mechanism of recruitment and regulation of several proteins with DNA methyltransferase activities, HAT and HDAC activities, and their roles in DNA replication and mRNA transcription have been reported. RNAi-mediated by miRNAs is also considered to be involved in epigenetics. The implications of epigenetics in diseases including cancer, cardiovascular disease, diabetes and neurological disorders have led to the understanding of their molecular pathology and disease management approaches. Inflammation is a well-established epigenetically-regulated biological process and the major genes involved are well described. There is a lack of sufficient evidence in the literature regarding the epigenetic regulation of gene expression in tendon tissues. Inflammation is a generalized occurrence associated with all tendinopathies, including rotator cuff insults. Conventional treatment strategies mainly deal with the management of pain and inflammation. However, the sustained disorganization of ECM increases the risk of recurrence of tendon damage. The possibilities of epigenetic regulation for the expression of inflammatory genes and the genes associated with tendon repair cannot be neglected. Additionally, extrapolation of the epigenetic changes in inflammation may offer opportunities to establish the same understanding in tendons.

Understanding the mechanism of DNA methylation patterns and histone modifications of tendon specific inflammatory genes can assist in improving current understanding of tendon pathology and may lead to the development of novel therapeutic/management strategies. The screening and validation of miRNA-mediated RNAi in tendon pathology can form another promising approach. Therapeutics based on miRNAs or miRNA-inhibitors possess appreciable therapeutic potential in several diseases, including cancer, however, none have been reported for rotator cuff tendons. Those miRNAs targeting multiple mRNAs in the same or different pathways can provide a promising approach, however, the selection of pathways and consideration of non-specific targets require careful evaluation. The mode of delivery of such therapeutic miRNAs is also a challenge. Further investigations are warranted in the field of tendon physiology/pathology for the elucidation of novel and promising epigenetic therapeutic targets and their clinical application.

\section{Acknowledgements}

Not applicable. 


\section{Funding}

This review was supported by a research grant from the State of Nebraska to DKA (grant no. LB506), grants from the National Heart, Lung and Blood Institute, National Institutes of Health (Bethesda, MD, USA; grant nos. R01 HL104516, R01 HL116042 and R01 HL120659) and from the George F. Haddix Faculty Grant from Creighton University (grant no. CU-240083).

\section{Availability of data and materials}

Not applicable.

\section{Authors' contributions}

FGT, CSB and DKA conceived the review and analyzed the relevant literature. FGT and CSB sourced the literature and wrote the first draft of the manuscript. FGT, MFD and DKA critically revised the manuscript. FGT and CSB produced the figures. All authors read and approved the final manuscript.

\section{Ethics approval and consent to participate}

Not applicable.

\section{Patient consent for publication}

Not applicable.

\section{Competing interests}

The authors confirm that they have no competing interests.

\section{References}

1. Thankam FG, Dilisio MF, Dougherty KA, Dietz NE and Agrawal DK: Triggering receptor expressed on myeloid cells and 5'adenosine monophosphate-activated protein kinase in the inflammatory response: A potential therapeutic target. Expert Rev Clin Immunol 12: 1239-1249, 2016.

2. Thankam FG, Dilisio MF and Agrawal DK: Immunobiological factors aggravating the fatty infiltration on tendons and muscles in rotator cuff lesions. Mol Cell Biochem 417: 17-33, 2016.

3. Page P: Shoulder muscle imbalance and subacromial impingement syndrome in overhead athletes. Int J Sports Phys Ther 6 : 51-58, 2011.

4. Waddington $\mathrm{CH}$ : Towards a theoretical biology. Nature 218: $525-527,1968$

5. Kornberg RD: Chromatin structure: A repeating unit of histones and DNA. Science 184: 868-871, 1974.

6. Weintraub $\mathrm{H}$ and Groudine M: Chromosomal subunits in active genes have an altered conformation. Science 193: 848-856, 1976.

7. Narlikar GJ, Fan HY and Kingston RE: Cooperation between complexes that regulate chromatin structure and transcription. Cell 108: 475-487, 2002.

8. Felsenfeld $\mathrm{G}$ and Groudine M: Controlling the double helix. Nature 421: 448-453, 2003

9. Surani MA, Hayashi K and Hajkova P: Genetic and epigenetic regulators of pluripotency. Cell 128: 747-762, 2007.

10. Human Genome Structural Variation Working Group; Eichler EE, Nickerson DA, Altshuler D, Bowcock AM, Brooks LD, Carter NP, Church DM, Felsenfeld A, Guyer M, et al: Completing the map of human genetic variation. Nature 447: 161-165, 2007.

11. Sebat J: Major changes in our DNA lead to major changes in our thinking. Nat Genet 39 (Suppl 7): S3-S5, 2007.

12. Jin B, Li Y and Robertson KD: DNA Methylation: Superior or subordinate in the epigenetic hierarchy? Genes Cancer 2: 607-617, 2011.
13. Herman JG and Baylin SB: Gene silencing in cancer in association with promoter hypermethylation. N Engl J Med 349: 2042-2054, 2003.

14. Voelter-Mahlknecht S: Epigenetic associations in relation to cardiovascular prevention and therapeutics. Clin Epigenetics 8: 4, 2016.

15. Shea JM, Serra RW, Carone BR, Shulha HP, Kucukural A, Ziller MJ, Vallaster MP, Gu H, Tapper AR, Gardner PD, et al: Genetic and epigenetic variation, but not diet, shape the sperm methylome. Dev Cell 35: 750-758, 2015

16. Lachner $M$ and Jenuwein T: The many faces of histone lysine methylation. Curr Opin Cell Biol 14: 286-298, 2002.

17. Pradhan S, Bacolla A, Wells RD and Roberts RJ: Recombinant human DNA (cytosine-5) methyltransferase. I. Expression, purification, and comparison of de novo and maintenance methylation. J Biol Chem 274: 33002-33010, 1999.

18. Leonhardt H, Page AW, Weier HU and Bestor TH: A targeting sequence directs DNA methyltransferase to sites of DNA replication in mammalian nuclei. Cell 71: 865-873, 1992.

19. Okano M, Bell DW, Haber DA and Li E: DNA methyltransferases Dnmt3a and Dnmt3b are essential for de novo methylation and mammalian development. Cell 99: 247-257, 1999.

20. Borgel J, Guibert S, Li Y, Chiba H, Schübeler D, Sasaki H, Forné $\mathrm{T}$ and Weber M: Targets and dynamics of promoter DNA methylation during early mouse development. Nat Genet 42: 1093-1100, 2010.

21. Wu H, Coskun V, Tao J, Xie W, Ge W, Yoshikawa K, Li E, Zhang Y and Sun YE: Dnmt3a-dependent nonpromoter DNA methylation facilitates transcription of neurogenic genes. Science 329: 444-448, 2010.

22. Ooi SK, Qiu C, Bernstein E, Li K, Jia D, Yang Z, Erdjument-Bromage H, Tempst P, Lin SP, Allis CD, et al: DNMT3L connects unmethylated lysine 4 of histone $\mathrm{H} 3$ to de novo methylation of DNA. Nature 448: 714-717, 2007.

23. Ramsahoye BH, Biniszkiewicz D, Lyko F, Clark V, Bird AP and Jaenisch R: Non-CpG methylation is prevalent in embryonic stem cells and may be mediated by DNA methyltransferase 3a. Proc Natl Acad Sci USA 97: 5237-5242, 2000.

24. Pinney S: Mammalian Non-CpG methylation: Stem cells and beyond. Biology (Basel) 3: 739-751, 2014.

25. Suzuki MM and Bird A: DNA methylation landscapes: Provocative insights from epigenomics. Nat Rev Genet 9: 465-476, 2008

26. Ehrlich M, Gama-Sosa MA, Huang LH, Midgett RM, Kuo KC, McCune RA and Gehrke C: Amount and distribution of 5-methylcytosine in human DNA from different types of tissues of cells. Nucleic Acids Res 10: 2709-2721, 1982.

27. Reik W, Dean W and Walter J: Epigenetic reprogramming in mammalian development. Science 293: 1089-1093, 2001.

28. Shen L, Kondo Y, Guo Y, Zhang J, Zhang L, Ahmed S, Shu J, Chen X, Waterland RA and Issa JP: Genome-wide profiling of DNA methylation reveals a class of normally methylated CpG island promoters. PLoS Genet 3: 2023-2036, 2007.

29. Henderson IR and Jacobsen SE: Tandem repeats upstream of the Arabidopsis endogene SDC recruit non-CG DNA methylation and initiate siRNA spreading. Genes Dev 22: 1597-1606, 2008.

30. Lienert F, Wirbelauer C, Som I, Dean A, Mohn F and Schübeler D: Identification of genetic elements that autonomously determine DNA methylation states. Nat Genet 43: 1091-1097, 2011.

31. Meissner A, Mikkelsen TS, Gu H, Wernig M, Hanna J, Sivachenko A, Zhang X, Bernstein BE, Nusbaum C, Jaffe DB, et al: Genome-scale DNA methylation maps of pluripotent and differentiated cells. Nature 454: 766-770, 2008

32. Smith ZD, Chan MM, Mikkelsen TS, Gu H, Gnirke A, Regev A and Meissner A: A unique regulatory phase of DNA methylation in the early mammalian embryo. Nature 484: 339-344, 2012.

33. Aran D, Sabato S and Hellman A: DNA methylation of distal regulatory sites characterizes dysregulation of cancer genes. Genome Biol 14: R21, 2013.

34. Sharif $O$ and Knapp S: From expression to signaling: Roles of TREM-1 and TREM-2 in innate immunity and bacterial infection. Immunobiology 213: 701-713, 2008.

35. Jones PA: Functions of DNA methylation: Islands, start sites, gene bodies and beyond. Nat Rev Genet 13: 484-492, 2012.

36. Maunakea AK, Nagarajan RP, Bilenky M, Ballinger TJ, D'Souza C, Fouse SD, Johnson BE, Hong C, Nielsen C, Zhao Y, et al: Conserved role of intragenic DNA methylation in regulating alternative promoters. Nature 466: 253-257, 2010. 
37. Laurent L, Wong E, Li G, Huynh T, Tsirigos A, Ong CT, Low HM, Kin Sung KW, Rigoutsos I, Loring J and Wei CL: Dynamic changes in the human methylome during differentiation. Genome Res 20: 320-331, 2010.

38. Mayer W, Niveleau A, Walter J, Fundele $R$ and Haaf $T$ : Demethylation of the zygotic paternal genome. Nature 403: 501-502, 2000

39. Inoue A and Zhang Y: Replication-dependent loss of 5-hydroxymethylcytosine in mouse preimplantation embryos. Science 334: 194, 2011

40. Guo JU, Su Y, Zhong C, Ming GL and Song H: Hydroxylation of 5-methylcytosine by TET1 promotes active DNA demethylation in the adult brain. Cell 145: 423-434, 2011.

41. Yang $\mathrm{H}$, Lin $\mathrm{H}, \mathrm{Xu} \mathrm{H}$, Zhang L, Cheng L, Wen B, Shou J, Guan K, Xiong Y and Ye D: TET-catalyzed 5-methylcytosine hydroxylation is dynamically regulated by metabolites. Cell Res 24: 1017-1020, 2014.

42. $\mathrm{Wu} \mathrm{H}$ and Zhang Y: Mechanisms and functions of Tet protein-mediated 5-methylcytosine oxidation. Genes Dev 25 2436-2452, 2011.

43. Kouzarides T: Chromatin modifications and their function. Cell 128: 693-705, 2007.

44. Gill G: SUMO and ubiquitin in the nucleus: Different functions, similar mechanisms? Genes Dev 18: 2046-2059, 2004.

45. Tsankova N, Renthal W, Kumar A and Nestler EJ: Epigenetic regulation in psychiatric disorders. Nat Rev Neurosci 8: 355-367, 2007.

46. Ragunathan K, Jih G and Moazed D: Epigenetic inheritance uncoupled from sequence-specific recruitment. Science 348 : 1258699,2015

47. Covic M, Hassa PO, Saccani S, Buerki C, Meier NI, Lombardi C, Imhof R, Bedford MT, Natoli G and Hottiger MO: Arginine methyltransferase CARM1 is a promoter-specific regulator of NF-kappaB-dependent gene expression. EMBO J 24: 85-96, 2005.

48. Tachibana M, Sugimoto K, Nozaki M, Ueda J, Ohta T, Ohki M, Fukuda M, Takeda N, Niida H, Kato H and Shinkai Y: G9a histone methyltransferase plays a dominant role in euchromatic histone $\mathrm{H} 3$ lysine 9 methylation and is essential for early embryogenesis. Genes Dev 16: 1779-1791, 2002.

49. Botuyan MV, Lee J, Ward IM, Kim JE, Thompson JR, Chen J and Mer G: Structural basis for the methylation state-specific recognition of histone $\mathrm{H} 4-\mathrm{K} 20$ by 53BP1 and Crb2 in DNA repair. Cell 127: 1361-1373, 2006.

50. Techima M: Inquiring for new approach to nursing education. III. A case in clinical practicum (2). Kango Kenkyu 24: 366-372, 1991 (In Japanese).

51. Fischle W, Tseng BS, Dormann HL, Ueberheide BM, Garcia BA, Shabanowitz J, Hunt DF, Funabiki H and Allis CD: Regulation of HP1-chromatin binding by histone $\mathrm{H} 3$ methylation and phosphorylation. Nature 438: 1116-1122, 2005.

52. Margueron R, Trojer P and Reinberg D: The key to development: Interpreting the histone code? Curr Opin Genet Dev 15: 163-176, 2005

53. Wysocka J, Swigut T, Xiao H, Milne TA, Kwon SY, Landry J, Kauer M, Tackett AJ, Chait BT, Badenhorst P, et al: A PHD finger of NURF couples histone H3 lysine 4 trimethylation with chromatin remodelling. Nature 442: 86-90, 2006.

54. Andrews FH, Gatchalian J, Krajewski K, Strahl BD and Kutateladze TG: Regulation of methyllysine readers through phosphorylation. ACS Chem Biol 11: 547-553, 2016.

55. Couture JF, Collazo E and Trievel RC: Molecular recognition of histone H3 by the WD40 protein WDR5. Nat Struct Mol Biol 13. 698-703, 2006

56. Kadota S and Nagata K: pp32, an INHAT component, is a transcription machinery recruiter for maximal induction of IFN-stimulated genes. J Cell Sci 124: 892-899, 2011

57. Shao Z, Raible F, Mollaaghababa R, Guyon JR, Wu CT, Bender W and Kingston RE: Stabilization of chromatin structure by PRC1, a Polycomb complex. Cell 98: 37-46, 1999.

58. Chandrasekharan MB, Huang F and Sun ZW: Ubiquitination of histone $\mathrm{H} 2 \mathrm{~B}$ regulates chromatin dynamics by enhancing nucleosome stability. Proc Natl Acad Sci USA 106: 16686-16691, 2009.

59. Canzonetta C, Vernarecci S, Iuliani M, Marracino C, Belloni C, Ballario P and Filetici P: SAGA DUB-Ubp8 deubiquitylates centromeric histone variant Cse4. G3 (Bethesda) 6: 287-298, 2015.

60. Sun L, Johnston SA and Kodadek T: Physical association of the APIS complex and general transcription factors. Biochem Biophys Res Commun 296: 991-999, 2002.
61. Muratani M and Tansey WP: How the ubiquitin-proteasome system controls transcription. Nat Rev Mol Cell Biol 4: 192-201, 2003.

62. Sun ZW and Allis CD: Ubiquitination of histone H2B regulates $\mathrm{H} 3$ methylation and gene silencing in yeast. Nature 418: 104-108, 2002.

63. Henry KW, Wyce A, Lo WS, Duggan LJ, Emre NC, Kao CF, Pillus L, Shilatifard A, Osley MA and Berger SL: Transcriptional activation via sequential histone $\mathrm{H} 2 \mathrm{~B}$ ubiquitylation and deubiquitylation, mediated by SAGA-associated Ubp8. Genes Dev 17: 2648-2663, 2003.

64. Gao C, Huang W, Kanasaki $\mathrm{K}$ and $\mathrm{Xu}$ Y: The role of ubiquitination and sumoylation in diabetic nephropathy. Biomed Res Int 2014: 160692, 2014.

65. Collins P, Mitxitorena I and Carmody R: The ubiquitination of $\mathrm{NF}-\kappa \mathrm{B}$ subunits in the control of transcription. Cells 5: pii: E23, 2016.

66. Moazed D: Small RNAs in transcriptional gene silencing and genome defence. Nature 457: 413-420, 2009.

67. Cech TR and Steitz JA: The noncoding RNA revolution-trashing old rules to forge new ones. Cell 157: 77-94, 2014.

68. Fire A, Xu S, Montgomery MK, Kostas SA, Driver SE and Mello CC: Potent and specific genetic interference by double-stranded RNA in caenorhabditis elegans. Nature 391: 806-811, 1998

69. Matzke M, Matzke AJ and Kooter JM: RNA: Guiding gene silencing. Science 293: 1080-1083, 2001.

70. Boosani C and Agrawal D: Epigenetic regulation of innate immunity by microRNAs. Antibodies 5: 8, 2016.

71. Castel SE and Martienssen RA: RNA interference in the nucleus: Roles for small RNAs in transcription, epigenetics and beyond. Nat Rev Genet 14: 100-112, 2013.

72. Kim VN, Han J and Siomi MC: Biogenesis of small RNAs in animals. Nat Rev Mol Cell Biol 10: 126-139, 2009.

73. Pak J and Fire A: Distinct populations of primary and secondary effectors during RNAi in C. elegans. Science 315: 241-244, 2007.

74. Halic M and Moazed D: Dicer-independent primal RNAs trigger RNAi and heterochromatin formation. Cell 140: 504-516, 2010.

75. Motamedi MR, Verdel A, Colmenares SU, Gerber SA, Gygi SP and Moazed D: Two RNAi complexes, RITS and RDRC, physically interact and localize to noncoding centromeric RNAs. Cell 119: 789-802, 2004.

76. Bühler M, Verdel A and Moazed D: Tethering RITS to a nascent transcript initiates RNAi- and heterochromatin-dependent gene silencing. Cell 125: 873-886, 2006

77. Holoch D and Moazed D: RNA-mediated epigenetic regulation of gene expression. Nat Rev Genet 16: 71-84, 2015.

78. Bayne EH, White SA, Kagansky A, Bijos DA, Sanchez-Pulido L Hoe KL, Kim DU, Park HO, Ponting CP, Rappsilber J and Allshire RC: Stc1: A critical link between RNAi and chromatin modification required for heterochromatin integrity. Cell 140: 666-677, 2010.

79. Gerace EL, Halic M and Moazed D: The methyltransferase activity of Clr4Suv39h triggers RNAi independently of histone H3K9 methylation. Mol Cell 39: 360-372, 2010.

80. Zaratiegui M, Castel SE, Irvine DV, Kloc A, Ren J, Li F, de Castro E, Marín L, Chang AY, Goto D, et al: RNAi promotes heterochromatic silencing through replication-coupled release of RNA Pol II. Nature 479: 135-138, 2011.

81. Li F, Martienssen R and Cande WZ: Coordination of DNA replication and histone modification by the Rik1-Dos2 complex. Nature 475: 244-248, 2011.

82. Millar NL, Murrell GA and McInnes IB: Inflammatory mechanisms in tendinopathy-towards translation. Nat Rev Rheumatol 13: 110-122, 2017

83. Campbell AL, Smith NC, Reilly JH, Kerr SC, Leach WJ, Fazzi UG, Rooney BP, Murrell GA and Millar NL: IL-21 receptor expression in human tendinopathy. Mediators Inflamm 2014: 481206, 2014.

84. Legerlotz K, Jones ER, Screen HR and Riley GP: Increased expression of IL- 6 family members in tendon pathology. Rheumatology (Oxford) 51: 1161-1165, 2012.

85. Dean BJ, Gettings P, Dakin SG and Carr AJ: Are inflammatory cells increased in painful human tendinopathy? A systematic review. Br J Sports Med 50: 216-220, 2016.

86. Thankam FG, Dilisio MF, Dietz NE and Agrawal DK: TREM-1, HMGB1 and RAGE in the shoulder tendon: Dual mechanisms for inflammation based on the coincidence of glenohumeral arthritis. PLoS One 11: e0165492, 2016

87. Guo J, Zhang JF, Li G and Chan KM: A Mini-review: MicroRNA in tendon injuries. J Stem Cell Res Ther 5: 303, 2015. 
88. Millar NL, Gilchrist DS, Akbar M, Reilly JH, Kerr SC, Campbell AL, Murrell GA, Liew FY, Kurowska-Stolarska M and McInnes IB: MicroRNA29a regulates IL-33-mediated tissue remodelling in tendon disease. Nat Commun 6: 6774, 2015.

89. Thankam FG, Boosani CS, Dilisio MF, Dietz NE and Agrawal DK: MicroRNAs associated with shoulder tendon matrisome disorganization in glenohumeral arthritis. PLoS One 11: e0168077, 2016

90.Thankam FG, Boosani CS, Dilisio MF and Agrawal DK: MicroRNAs associated with inflammation in shoulder tendinopathy and glenohumeral arthritis. Mol Cell Biochem 437: 81-97, 2018.

91. Medzhitov R and Horng T: Transcriptional control of the inflammatory response. Nat Rev Immunol 9: 692-703, 2009.

92. Wierda RJ, Geutskens SB, Jukema JW, Quax PH and van den Elsen PJ: Epigenetics in atherosclerosis and inflammation. J Cell Mol Med 14: 1225-1240, 2010.

93. De Santa F, Totaro MG, Prosperini E, Notarbartolo S, Testa G and Natoli G: The histone H3 lysine-27 demethylase Jmjd3 links inflammation to inhibition of polycomb-mediated gene silencing. Cell 130: 1083-1094, 2007.

94. Hahn MA, Hahn T, Lee DH, Esworthy RS, Kim BW, Riggs AD, Chu FF and Pfeifer GP: Methylation of polycomb target genes in intestinal cancer is mediated by inflammation. Cancer Res 68 : 10280-10289, 2008.

95. Chen X, El Gazzar M, Yoza BK and McCall CE: The NF-kappaB Factor RelB and Histone H3 lysine methyltransferase G9a directly interact to generate epigenetic silencing in endotoxin tolerance. J Biol Chem 284: 27857-27865, 2009.

96. Gazzar ME, Yoza BK, Chen X, Hu J, Hawkins GA and McCall CE: G9a and HP1 couple histone and DNA methylation to TNF transcription silencing during endotoxin tolerance. J Biol Chem 283: 32198-32208, 2008.

97. Villagra A, Sotomayor EM and Seto E: Histone deacetylases and the immunological network: Implications in cancer and inflammation. Oncogene 29: 157-173, 2010.

98. Bayarsaihan D: Epigenetic Mechanisms in Inflammation. J Dent Res 90: 9-17, 2011.

99. Shuto T, Furuta T, Oba M, Xu H, Li JD, Cheung J, Gruenert DC, Uehara A, Suico MA, Okiyoneda T and Kai H: Promoter hypomethylation of Toll-like receptor- 2 gene is associated with increased proinflammatory response toward bacterial peptidoglycan in cystic fibrosis bronchial epithelial cells. FASEB J 20 782-784, 2006.

100. Takahashi N: Microbial ecosystem in the oral cavity: Metabolic diversity in an ecological niche and its relationship with oral diseases. Int Congr Ser 1284: 103-112, 2005.

101. Brand RA: Surgical anatomy of the rotator cuff and the natural history of degenerative periarthritis. Clin Orthop Related Res 466: 543-551, 2008 .
102. Claycombe KJ, Brissette CA and Ghribi O: Epigenetics of inflammation, maternal infection, and nutrition. J Nutr 145 (Suppl): 1109S-1115S, 2015

103. Niwa T, Tsukamoto T, Toyoda T, Mori A, Tanaka H, Maekita T, Ichinose $\mathrm{M}$, Tatematsu $\mathrm{M}$ and Ushijima $\mathrm{T}$ : Inflammatory processes triggered by Helicobacter pylori infection cause aberrant DNA methylation in gastric epithelial cells. Cancer Res 70 1430-1440, 2010.

104. Ding W, Mouzaki M, You H, Laird JC, Mato J, Lu SC and Rountree CB: CD133+ liver cancer stem cells from methionine adenosyl transferase 1A-deficient mice demonstrate resistance to transforming growth factor (TGF)-beta-induced apoptosis. Hepatology 49: 1277-1286, 2009.

105. Wang YQ, Li YM, Li X, Liu T, Liu XK, Zhang JQ, Guo JW, Guo LY and Qiao L: Hypermethylation of TGF- $\beta 1$ gene promoter in gastric cancer. World J Gastroenterol 19: 5557-5564, 2013.

106. Boosani CS, Dhar K and Agrawal DK: Down-regulation of hsa-miR-1264 contributes to DNMT1-mediated silencing of SOCS3. Mol Biol Rep 42: 1365-1376, 2015.

107. Cheng C, Huang C, Ma TT, Bian EB, He Y, Zhang L and Li J: SOCS1 hypermethylation mediated by DNMT1 is associated with lipopolysaccharide-induced inflammatory cytokines in macrophages. Toxicol Lett 225: 488-497, 2014.

108. Kuo PL, Shen KH, Hung SH and Hsu YL: CXCL1/GROa increases cell migration and invasion of prostate cancer by decreasing fibulin-1 expression through NF- $\kappa \mathrm{B} / \mathrm{HDAC} 1$ epigenetic regulation. Carcinogenesis 33: 2477-2487, 2012.

109. Chen S, Boosani C and Agrawal DK: Abstract 12818: KPNA4 mediates vitamin D-dependent inhibition of NF- $\mathrm{B}$ activity in swine epicardial preadipocytes. Circulation 130: A12818, 2014.

110. Cao B, Yang Y, Pan Y, Jia Y, Brock MV, Herman JG and Guo M: Epigenetic silencing of CXCL14 induced colorectal cancer migration and invasion. Discov Med 16: 137-147, 2013.

111. Ramos EA, Grochoski M, Braun-Prado K, Seniski GG, Cavalli IJ, Ribeiro EM, Camargo AA, Costa FF and Klassen G: Epigenetic changes of CXCR4 and its ligand CXCL12 as prognostic factors for sporadic breast cancer. PLoS One 6: e29461, 2011.

112. Yasmin R, Siraj S, Hassan A, Khan AR, Abbasi R and Ahmad N: Epigenetic regulation of inflammatory cytokines and associated genes in human malignancies. Mediators Inflamm 2015: 201703, 2015 .

This work is licensed under a Creative Commons Attribution-NonCommercial-NoDerivatives 4.0 International (CC BY-NC-ND 4.0) License. 